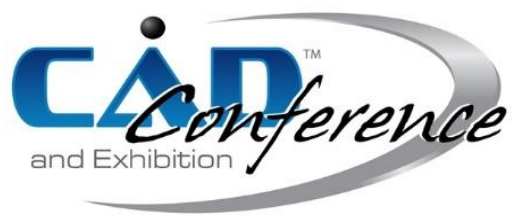

Title:

Recognition and Simplification of Holes in CAD Models of an Injection Mold for Mold Flow Analysis

Authors:

Jiing-Yih Lai, jylai@ncu.edu.tw, National Central University, Zhongli, Taiwan

Pei-Pu Song, scarlet811025@gmail.com, National Central University, Zhongli, Taiwan

Chia-Hsiang Hsu, davidhsu@moldex3d.com, CoreTech System (Moldex3D) Co., Ltd., Hsinchu, Taiwan

Yao-Chen Tsai, oncloud@gmail.com, CoreTech System (Moldex3D) Co., Ltd., Hsinchu, Taiwan

Keywords:

Hole Recognition, Hole Simplification, Feature Recognition, Feature Suppression, B-rep Model

DOI: 10.14733/cadconfP.2019.1-7

Introduction:

In mold flow analysis, it is necessary to convert the computer-aided design (CAD) models of an injection mold into solid meshes during the analysis. The main components of an injection mold that need to be modeled are the core, cavity, runner system, cooling channels, and the mold base. Traditionally, a mold base is modeled as a rectangular box to simplify the mold structure. However, in some applications, it is necessary to consider the non-simplified, original plates of the mold base in order to increase the accuracy of the mold flow analysis. As more components of the mold are involved and meshed, the total number of mesh nodes increases. Therefore, mesh reduction becomes an important issue to study.

Typical mold plates on a mold base are clamping plates, cavity plates, stripper plates, core plates, support plates, spacer blocks, locating plates, and ejector clamping plates. Holes are a common feature in these components. If all holes are preserved and meshed, then, because the mesh size near holes should be smaller, a tremendous amount of nodes would be generated. On the contrary, if most holes on mold plates are simplified, then the total amount of nodes can be substantially reduced. It can be noted that the simplification of holes on most mold plates would not affect the results of the analysis because they are assembled with counterparts during the injection process.

On the other hand, holes that need to be preserved in mold flow analyses sometimes go across different CAD models. For example, a cooling channel is usually composed of a series of holes passing through different mold plates. Similarly, a runner system usually starts from the top plate, passes through a stripper or support plate, and finally reaches the core and cavity. Although hole recognition modules are available in some CAD systems, most of them focus on individual CAD models only; when a set of CAD models representing an injection mold are considered, each of them must be processed, one by one. To facilitate the automation of the CAD pre-processing process in mold flow analysis, it is necessary to detect and record all related holes across different CAD models, rather than within each CAD model only.

In feature recognition, most investigations employ the topological relationship of adjacent entities for the recognition of features. A method has been proposed, in accordance with the topological relationship of the boundary representation (B-rep) model, to solve the problem that the boundary of the holes is not filleted when using the attributed adjacency graph (AAG) method [3]. This proposed method utilizes the property that a hole, in the B-rep model for hole recognition, is always accompanied with an inner loop. However, the types of holes that can be recognized are limited. Song et al. [7] presented a method for the recognition and suppression of different kinds of holes on a mold base. An approach based on the loop data of the B-rep model was developed to search for holes on each CAD model. Holes that are connected in series on the same CAD model were also detected and recorded. 
Finally, a hole suppression algorithm was proposed to eliminate each set of connected holes. When a hole is filleted on its boundary, it may be necessary to recognize the fillet first, and then perform the recognition of the hole in terms of the fillet information. Several fillet recognition algorithms are available in literature [1], [2], [4], [8].

\section{Main Idea:}

To aid the analysis, holes can be classified into several types. A single hole exists alone, such as the cases in Fig. 1(a) and (b). Several holes that connect to each other can form different hole structures. If all holes connect to each other in series, it is called a ladder hole, such as the cases in Fig. 1(c) and (d), which contain two and three holes connected in series, respectively. If the hole breaks through the surface of the part it is called a "through hole", otherwise it is called a "blind hole". Holes in a ladder hole generally can be simplified simultaneously. If several holes inside a larger hole are arranged in parallel, such as the case in Fig. 1(e), then it is called a parallel hole structure. Hole simplification in this structure should be considered case by case. Related holes may be across different mold parts, such as the case in Fig. 1(f), each of which could be either a single hole or a ladder hole on CAD models.

A hole is essentially composed of three types of

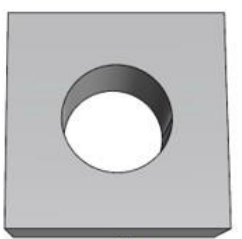

(a)

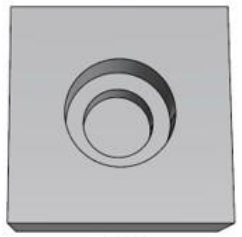

(d)

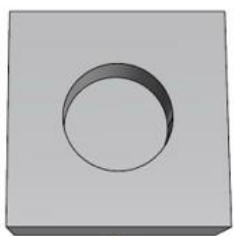

(b)

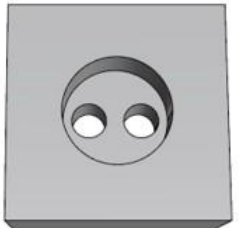

(e)

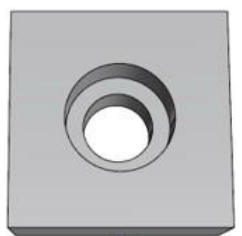

(c)

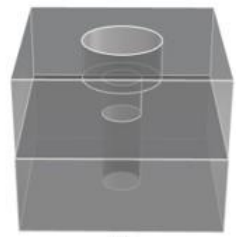

(f)

Fig.1: Classification of hole, (a) single-through hole, (b) single-blind hole, (c) ladder-through hole, (d) ladderblind hole, and (e) parallel hole structure, and (f) related holes across different components. faces, namely base, side, and bottom faces. A base face is a face where a hole resides; the hole can form an inner loop on the base face. A bottom face is at the bottom of a hole. The side face is a face that connects to both base and bottom faces simultaneously. The angle between the side and base faces is always convex. A blind hole has only one loop and the angle between the side and bottom faces is concave. The base and bottom faces of a blind hole can easily be distinguished. A through hole have two loops and the angles of the faces on both loops are convex. As both angles are convex, the face that is adjacent to the input loop is regarded as the base face, while the face that is adjacent to the second loop is regarded as the bottom face. The proposed hole recognition and simplification method is divided into four main phases: (1) hole recognition on each CAD model, including single and ladder holes, (2) recognition of related holes across different CAD models, (3) detection of holes that should be preserved, and (4) simplification of unnecessary holes. A detailed description for each of these steps is given below.

\section{Hole Recognition on each CAD Model}

Holes on each CAD model include single and ladder holes. The composition of the faces for each hole is evaluated first, and then holes that connect to each other in series are evaluated and formed as ladder holes. The procedure for this algorithm is shown in Fig. 2, in which three types of holes, with different end faces and hole structures, are illustrated. The inputs are the loop and fillet data (see Fig. 2(a)) on each CAD model and the outputs are the composition of the faces for each hole and the topological data of the ladder holes. This algorithm can be divided into three steps.

First, the facial composition is determined for each hole. The hole search is performed loop by loop. For each loop, the base face that it resides on is found. If all edges on this loop are convex, then a hole exists and all the neighboring faces are recorded. If the neighboring face is not a fillet, then it is considered as a side face. In contrast, if it is a fillet, then the neighboring face of this fillet is considered as a side face. Once all side faces are obtained, their neighboring face can be found. Similarly, if the neighboring face is not a fillet, then it is regarded as a bottom face. In contrast, if it is a fillet, then the neighboring face of this fillet is regarded as a bottom face. Fig. 2(b) shows that four holes corresponding to the case in Fig. 2(a) are detected. 
Second, each hole is evaluated as either through or blind. Once all holes are obtained, each hole is checked, one by one, to attribute it as either blind or through. If all edges between the side and bottom faces are concave, then it is a blind hole. If all edges between the side and bottom faces are convex, then it is a through hole. It should be noted that a through hole is counted twice, as the loops on both of its end faces are checked individually. Therefore, repeated holes should be detected and removed. Fig. 2(c) shows that two blind holes and two through holes are detected.

Third, related holes on each CAD model are detected. The relationship between two neighboring holes is obtained by recording the indices of faces that are shared bilaterally. Several holes that connect to each other in series can be regarded as a ladder hole. The cross section of a ladder hole is similar to that of a ladder, which is why it is termed a ladder hole. A ladder hole is composed of a series of holes

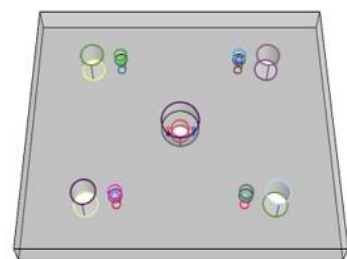

(a)

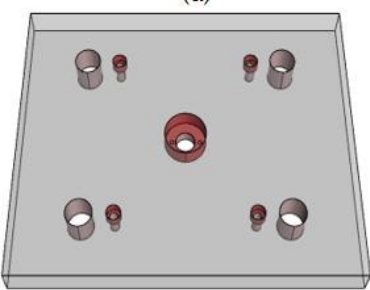

(c)

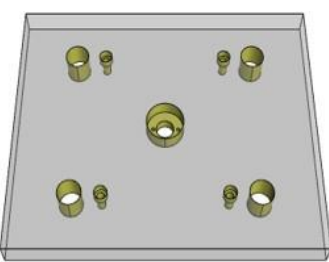

(b)

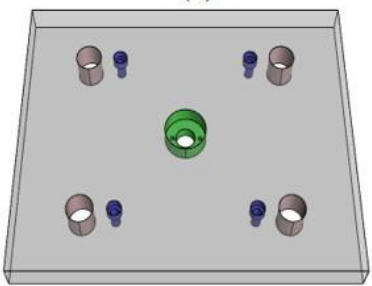

(d)

Fig. 2: Hole recognition procedures: (a) input loop data (each color denotes a loop), (b) search face composition (yellow) of each hole, (c) compute type and associated properties of each hole (pink: through hole, red: blind hole), and (d) detect related holes (blue: ladder hole, green: parallel hole structure).

connected in sequence, with each of the holes called a layer; the first hole is the first layer, the second hole is the second layer, etc.

\section{Recognition of Related Holes across Different CAD Models}

When several CAD models representing different mold plates are input to a CAD system, no topological relationship exists between these CAD models. To detect related holes across different CAD models, it is necessary to establish the relationships between CAD models that are in contact. This study assumes that the mold set is well aligned such that its three main directions are parallel to $x, y$ and $z$ directions, respectively. In addition, its parting direction is along the $z$ direction. If the CAD model of a mold does not follow this assumption, a pre-adjustment of its orientation is necessary.

As Fig. 3 depicts, the proposed algorithm is

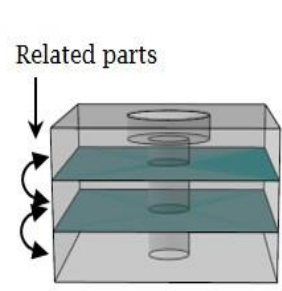

(a)

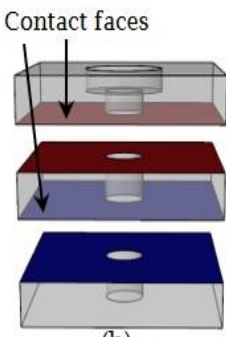

(b)

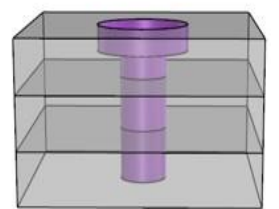

(c)

Fig. 3: Procedures of detecting related holes across different parts: (a) generate the relationship between parts, (b) search the contact faces, and (c) generate the relationship of holes across different parts.

divided into four steps. First, the mold plates are sorted by using the parting direction: the order of the mold plates in the CAD system is determined. Second, the relationships among the plates are generated (Fig. 3(a)). All CAD models that are in contact with each other are detected by checking the intersection of their bounding boxes. Third, the contact faces are searched for (Fig. 3(b)): the contact faces of two adjacent CAD models can be used to evaluate the holes passing across different CAD models. Finally, the relationship between the holes passing across different plates is generated (Fig. 3(c)). For holes passing across different CAD models, each of the holes can generate a loop on the corresponding contact face. By checking the loops on a pair of contact faces from each of the two adjacent CAD models, the holes passing across different CAD models can be detected. A detailed description for each of the proposed steps in the recognition algorithm is given below.

Sort the Mold Plate by Using the Parting Direction: When the mold plate CAD models are input to a $\mathrm{CAD}$ system, no order exists among these CAD models in the data structure. However, they should be sequentially arranged for subsequent steps. This is done by calculating the order of each mold plate by 
using the parting direction. The parting direction is a parameter determined by the user, with the default being the positive $\mathrm{z}$ direction.

Generate the Relationship among Plates: A simple and computationally efficient method, which employs the bounding boxes of all CAD models, was developed to evaluate adjacent CAD models. The bounding box of a CAD model is represented by two marginal points, $P_{\min }=\left(X_{\min }, y_{\min }, Z_{\min }\right)^{T}$ and $P_{\max }=\left(X_{\max }\right.$, $\left.y_{\max }, Z_{\max }\right)^{T}$. An algorithm that compares the $\mathrm{x}, \mathrm{y}$, and $\mathrm{z}$ coordinates of two sets of marginal points was developed to check the intersection of two bounding boxes. If two CAD models intersect, then the region of intersection can be represented as a range box which is essentially another bounding box. In contrast, if two CAD models do not intersect, then no range box is found. If two CAD models are in contact at a plane, then the range box is reduced to a plane where the minimum and maximum coordinate values along the $\mathrm{x}, \mathrm{y}$, or $\mathrm{z}$ axis are equal. In the proposed procedure, the bounding boxes of all CAD models are checked pair by pair.

Search for Contact Faces: The range box of two adjacent CAD models is then employed to search for contact faces. First, a set of candidate contact faces from each CAD model is evaluated. The bounding box of each face on a CAD model is compared with the range box. If they intersect, then the corresponding face is inside the range box. Two sets of candidate contact faces will be obtained, one from each CAD model. The contact situation of each pair of candidate contact faces can be divided into the following two cases: (1) they contact at one edge: it occurs at two adjacent holes or two adjacent sides faces of different mold plates; (2) they contact at a surface: two candidate contact faces are faceto-face contact. Second, the faces on both sets of candidate contact faces are compared one by one to find the contact faces. Among the candidate contact faces in step (1), case (2) is what we need, whereas case (1) is skipped. In the proposed procedure, the faces on both sets of candidate faces are checked pair by pair.

Generate the Relationship between Holes across Different Plates: At this stage, holes on each CAD model have already been recognized and the ladder holes have been grouped. The main issue here is to generate the relationship between holes on each pair of contact faces. All groups of holes passing across different CAD models can thus be integrated and arranged in sequence. The proposed process is divided into two main steps. First, the holes on the contact faces are located. Each contact face is essentially a base face or bottom face of a hole. Therefore, the holes that are located on each contact face can be obtained by searching the hole dataset. After the first step, the two sets of holes corresponding to a pair of contact faces are obtained. Each set of holes is located on one of the contact faces. Second, coaxial holes are searched for across different CAD models and grouped together. For the two sets of holes corresponding to the same pair of contact faces, coaxial holes are regarded as a group. The above process is implemented for all pairs of contact faces.

Detection of Holes That Should Be Preserved

In mold flow analyses, holes on the core, cavity, runner system, and cooling channels should be preserved, as they are included in the mesh model. Holes on the core, cavity, and runner system can easily be preserved as they belong to part of the product. However, cooling channels are embedded inside the mold, and cannot be recognized by using the abovementioned hole recognition method. To solve this problem, the following geometric properties of cooling channels should be

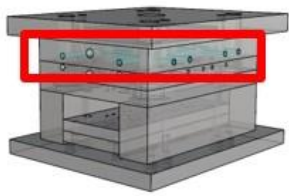

(a)

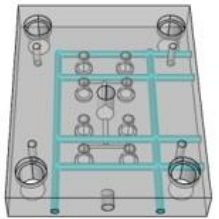

(b)

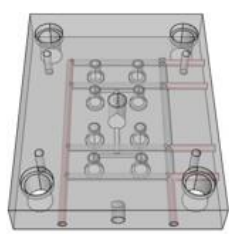

(c)

Fig. 4: Geometric properties of cooling channels on a mold, (a) entries and exits are located on side faces of the mold, (b) contain multiple tubes intersected each other, and (c) not all tubes are exposed to the part surface

considered. Firstly, a cooling channel has a circular cross section, and is exposed to the part surface at its entry and exit, as shown in Fig. 4(a). Secondly, a cooling channel can either be a straight tube passing through a mold plate, or the intersection of multiple tubes passing through one or several mold plates, as shown in Fig. 4(b). If it is a straight tube, then the tube can be recognized by using the abovementioned hole recognition method. However, if it contains multiple tubes that intersect each other, only the tubes exposed to the part surface can be recognized, whereas the others embedded inside the mold cannot be recognized, as shown in Fig. 4(c). The recognition of cooling channels is divided into 
three steps: (1) computing the intersected tubes, (2) computing the side faces of the mold, and (3) searching for cooling channels.

Computing Intersected Tubes: The cylindrical face of a tube can either be composed of one or two faces. Therefore, cylindrical faces of the same tube should be grouped first. For all cylindrical faces, three rules are tested: (1) two cylindrical faces must be adjacent to each other through their linear edges, (2) for both cylindrical faces, each surface normal must be the inverse of its radial direction, and (3) the cross-sectional edges of two cylindrical faces must be able to form a closed circle. If all three rules are satisfied, then these two cylindrical faces belong to the same tube, and hence are grouped. The procedure to compute the intersected tubes can then be performed. Starting from a recognized through hole, its bottom face is checked if it belongs to a group of cylindrical faces. If it does not, then this hole is skipped and the next through hole is selected. If it does, then the neighboring group of cylindrical faces is searched for, starting from this group of cylindrical faces. This search is continued until no more neighboring groups of cylindrical faces are found. All neighboring cylindrical faces obtained in this step are recorded as a set of intersected tubes.

Computing Side Faces of the Mold: The side faces of a mold are evaluated by using the contact faces obtained previously. For each contact face, its outer loop is employed to search for adjacent faces. The surface normal is checked to see if each adjacent face is the same as that of the bounding plane. If it is the same, then this adjacent face is a side face.

Searching for Cooling Channels: As the entries and exits of a cooling channel are located on the side faces of a mold, any entry or exit hole can be found and regarded as a seed hole to search for the entire cooling channel. While not all holes on the side faces belong to cooling channels, entry or exit holes can be distinguished by their property of being both a through hole and an intersected hole. Once the seed hole is obtained, the entire cooling channel can be obtained simply by searching for the neighboring faces, one by one.

\section{Hole Simplification}

Hole simplification in a Brep model involves essentially changing the faces and associated entities related to the hole to remove the hole itself. As a hole is essentially composed of three kinds of faces, the base, side, and bottom faces, when a hole is removed, some of its faces are removed from

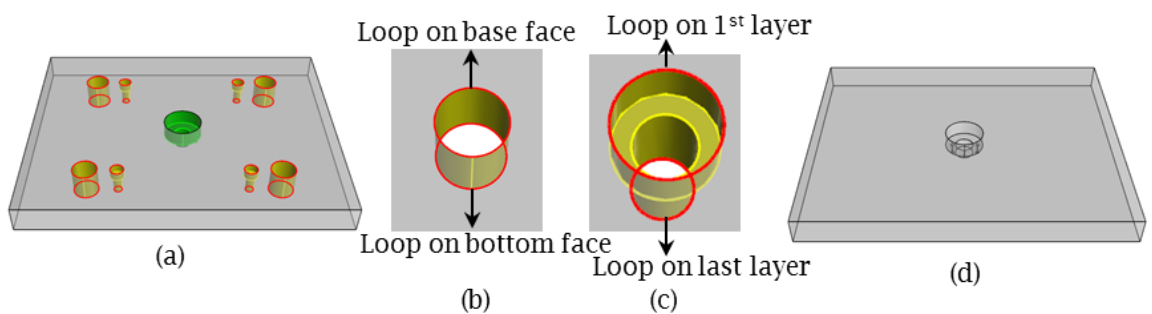

Fig. 5: Example of hole simplification: (a) a mold plate before hole simplification, (b) faces to manipulate for a through hole, (c) faces to manipulate for a ladder-through hole, and (d) the mold plate after hole simplification.

the data structure, whereas the others are modified. Take the mold plate shown in Fig. 5(a) as an example, which includes three kinds of holes: runner, through and ladder-through holes. The runner hole is preserved. For a through hole, as shown in Fig 5(b), the base faces on both sides are modified by removing their inner loops, whereas the side face is removed. For a ladder-through hole, as shown in Fig. 5(c), the base and bottom faces of the first and last layers, respectively, are modified, whereas all other faces are removed. For the through hole and the ladder-through hole, the associated loops, edges, trims, and vertices in the B-rep data are either deleted or modified too. The sample model after the hole simplification was performed is shown in Fig. 5(d).

Holes are divided into two types, single or ladder, and then further be subdivided into either blind or through. The faces that should be modified or removed for each type are: (1) single-through holes: the base and bottom faces are modified, while the side face is removed; (2) single-blind holes: the base face is modified, while the side and bottom faces are removed; (3) ladder-through holes: the base and bottom faces of the first and last layers, respectively, are modified, while all other faces are removed; (4) ladder-blind holes: the base face of the first layer is modified, while all other faces are removed.

The operations required for the modification and removal of each face are similar to the case shown in Fig. 5. That is, when a face is modified, its inner loop and associated entities are deleted from the B- 
rep data. In addition, when a face is removed, the face and associated entities are deleted from the Brep data. For cases (1) and (2), each of the single holes is individually simplified. However, for cases (3) and (4), all holes of a ladder hole can be removed either completely or partially. For partial removal, the lower layers of holes should be removed while the upper layers of holes are preserved. Such an operation can be done manually.

\section{Results and Discussion}

We tested the feasibility of the proposed algorithms with a program written in $\mathrm{C}++$ and based on the Rhino CAD platform [7] and the openNURBS functions [6]. The proposed algorithm is only compatible with CAD models with manifold topology and geometry. Fig. 6 illustrates the hole recognition and simplification results for three injection molds, where Fig. 6(a), (b), and (c) denote the original CAD models, hole recognition, and hole

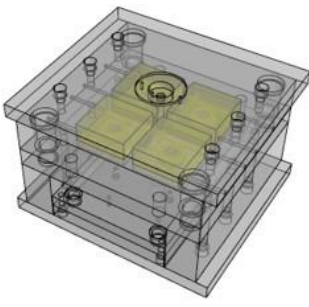

(a)

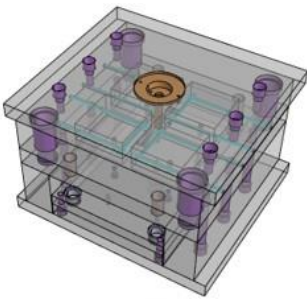

(b)

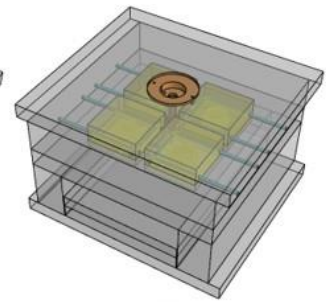

(c)

Fig. 6: Three injection molds used in this study, (a) the original CAD models, (b) hole recognition, and (c) hole simplification. simplification, respectively. Each example contains all CAD models of a mold set, including many different kinds of mold plates, core and cavity. Most of the holes are exposed to part surfaces, except for the cooling channels, which are embedded inside the mold plates. The holes recognized on the mold plates are classified as the runner, cooling channels, single-blind, single-through, ladder-blind, or ladderthrough. The runner and cooling channels are in green and water blue, respectively, while each type of the other holes are also in different colors, as shown in Fig. 6(b). Only the sprue and cooling channels were preserved, while all other holes on the mold plates were removed, as shown in Fig. 6(c).

\section{Conclusions:}

The development of hole recognition and simplification algorithms for injection molds was studied, and the feasibility of the proposed algorithms by using several mold sets was verified. After hole recognition, it divided all holes into two groups, either the through hole or the blind hole types. Related holes were divided into either the ladder hole or parallel hole structure, with the hole type determined for each hole within all the CAD models. Coaxial holes passing across different CAD models were then evaluated. Finally, because they should be preserved, holes related to the runner and cooling channels were individually detected. Holes that should be deleted were divided into five types: ladder holes passing across different CAD models, ladder holes contained within a CAD model, parallel hole structures, singlethrough holes, and single-blind holes. For each hole type, the faces that should be deleted or modified, and their associated entities that should be processed, were analyzed. The operations required for the hole simplification were also addressed. The proposed method can preserve the runner and cooling channels, while deleting all other holes in the mold set. In cases where it is necessary to consider the original, non-simplified mold plates of a mold base (typically modeled as a rectangular box for simplification) in a mold flow analysis, the total amount of mesh nodes can be substantially reduced by using the proposed method.

\section{References:}

[1] Cui, X.; Gao, S.: Zhou, G.: An efficient algorithm for recognizing and suppressing blend features, Computer-Aided Design and Applications, 1(1-4), 2004, 421-428. http://dx.doi.org/10.1080/ 16864360.2004 .10738284

[2] Li, B.; Liu J.: Detail feature recognition and decomposition in solid model, Computer Aided Design, 34(5), 2002, 405-414. http://dx.doi.org/10.1016/S0010-4485(01)00118-X

[3] Li, J.; Sun, L.; Peng, J.; Du, J.; Fan, L.: Automatic small depression feature recognition from solid Brep models for meshing, 2011 International Conference on Electrical and Control Engineering, 2011, 4386-4389. http://dx.doi.org/10.1109/ICECENG.2011.6057432 
[4] Li, J.; Tong, G.; Shi, D.; Geng, M.; Zhu, H.; Hagiwara, I.: Automatic small blend recognition from Brep models for analysis, Engineering with Computers, 25(3), 2009, 279-285. https://doi.org/10.1007/s00366-009-0127-4

[5] openNURBS, http://www.rhino3d.com/tw/opennurbs

[6] Rhinoceros, http://www.rhino3d.com

[7] Song, P.-P.; Lai, J.-Y.; Tsai, Y.-C.; Hsu, C.-H.: Automatic recognition and suppression of holes on mold bases for finite element applications, Engineering with Computers, 2018, 1-20. https://doi.org/10.1007/s00366-018-0640-4

[8] Venkataraman, S.; Sohoni, M: Blend recognition algorithm and applications, The sixth ACM Symposium on Solid Modeling and Applications, 2001, 99-108. http://dx.doi.org/10.1145/ $\underline{376957.376970}$ 